\title{
Yield and Quality Based Phenotyic Evaluation of Germplasm of Brinjal (Solanum melongena L.) Under Semi-Arid Conditions
}

\author{
Shiwangi Srivastava ${ }^{1}$, P. Saidaiah ${ }^{2 *}$, N. Shivraj ${ }^{3}$ and K. Ravinder $\operatorname{Reddy}^{1}$ \\ ${ }^{1}$ Department of Vegetable Science, College of Horticulture, Sri Konda Laxman Telangana \\ State Horticulture University, Rajendranagar, Hyderabad-500030, Telangana, India \\ ${ }^{2}$ Department of Genetics and Plant breeding, SKLTSHU, Rajendranagar, Hyderabad-500030, \\ Telangana, India \\ ${ }^{3}$ Economic Botany, NBPGR Regional Station, Rajendranagar, Hyderabad-500030, \\ Telangana, India \\ *Corresponding author
}

\section{A B S T R A C T}

A field investigation as carried out with 35 genotypes of brinjal at Department of Vegetable Science, SKLTSHU, Rajendranagar, Hyderabad during Rabi, 2017-18. The RBD ANOVA revealed highly significant differences among the genotypes for all the traits indicating the presence of sufficient variability in the experimental material.

\section{Keywords}

Eggplant [Solanum melongena $\mathrm{L}$.$] , field$ investigation

Article Info

Accepted: 07 June 2019 Available Online: 10 July 2019 Selection of suitable parents is an important criterion for success of crop improvement program. Mean performance of germplasm under study showed that the IC-136148 recorded the highest plant height $(104.19 \mathrm{~cm})$, number of fruits per plant $(26.33)$, average fruit weight $(0.19 \mathrm{~kg})$, fruit yield per plant $(3.07 \mathrm{~kg})$ and total phenol content $(59.23 \mathrm{mg} /$ $100 \mathrm{~g})$. The genotype IC-136546 recorded the highest number of branches per plant and IC136237 for days to first flowering (36.55 days) and days to 50 percent flowering (37.67 days) were also reported. The genotype, IC-136096 recorded the highest number of flower clusters per plant (24.27). The highest number of fruits per cluster of 2.50 and days to last harvest (157.67) was recorded by the genotype IC-136231. The genotype IC-136311 recorded less number of days for first harvest (51.67days), IC-136196 for fruit length $(16.90 \mathrm{~cm})$ and IC-136184 registered highest values for fruit width $(6.49 \mathrm{~cm})$. The highest ascorbic acid content of $7.44 \mathrm{mg} / 100 \mathrm{~g}$ was recorded with the genotype IC-136176. The genotype, IC-136309 in 12.93 percent was found to be tolerant to shoot and fruit borer infestation. The germplasm identified in the present study can be suitable utilized for varietal release and as parents in breeding programmes for further improvement of the traits.

\section{Introduction}

Brinjal or Aubergine or Eggplant [Solanum melongena $\mathrm{L} . \quad(2 \mathrm{n}=2 \mathrm{x}=24)$ ] belongs to the family Solanaceae which is one of the most important commercial vegetable crops in the world, especially in the tropics and subtropics (Kalloo, 2002). The brinjal is of much importance in the warm areas of Far East, being grown extensively in India, and other 
Asian countries like Bangladesh, Pakistan, and Philippines. The cultivated brinjal is of Indian origin and has been in cultivation for long time. Now, India is considered as centre of origin and diversity of brinjal (Vavilov, 1951; Isshiki et al., 1994).

Brinjal is rich source of Anthocyanins, Vitamin-C and phenolic compounds, which are powerful antioxidants (Vinson et al., 1998). It quite high in nutritive value as compared with tomato (Choudhary, 1976), it is an important source of carbohyderate $(4.0 \mathrm{~g})$, protein $(1.4 \mathrm{~g})$, fibre $(1.3 \mathrm{~g})$, vitamin A (124 IU), phosphorus (47 mg), potassium (2.0mg) and iron $(0.3 \mathrm{mg})$ Besides that, it contains alkaloid solanine in roots and leaves (Dhankhar and Singh, 1984). Dry fruits are reported to contain goitrogenic principles. Bitterness in brinjal is due to the presence of glycoalkaloids. The peel of brinjal has significant amounts of anthocyanin with antioxidant activity and protects against cancer, ageing, inflammation and neurological diseases (Hanur, 2011). Being the centre of origin, India has accumulated with wide range of variability in this crop but still India is the second largest producer of brinjal after China with an area and production of 0.71 million hectare and 13.5 million tonnes, respectively (NHB, 2015). Therefore collection and evaluation of genotypes is a pre-requisite for genetic improvement of the crop. Keeping the above in view, the present investigation was carried out to study the phenotypic performance of brinjal germplasm.

\section{Materials and Methods}

The study was carried out at the PG Research Block, Department of Vegetable Science, Sri Konda Laxman Telangana State Horticultural University, Rajendranagar, Hyderabad during rabi, 2017-18. The experiment was laid out with thirty five genotypes of brinjal in Randomized Block Design (RBD) with three replications. The genotypes tested consisted of the lines collected from different localities over states by NBPGR. The seeds of 35 genotypes were sown in nursery. Twenty six days old seedlings were transplanted to the main field. In each replication, each genotype was grown in a three row plot accommodating 21 plants with row-to-row spacing of $60 \mathrm{~cm}$ and plant-to-plant spacing of $45 \mathrm{~cm}$. The recommended package of practices was followed. Necessary plant protection measures were carried out uniformly to safe guard the germplasm lines. List of brinjal genotypes used in the experiment are presented in Table 1. Five randomly chosen plants in each replication of each entry were labelled and used for recording the observations for the growth parameters.

The data on fourteen quantitative, two qualitative and three pest \& disease incidence characters were recorded on five competitive and randomly selected plants in each genotype and in each replication i.e., plant height $(\mathrm{cm})$, number of branches per plant, number of flower clusters per plant, number of flowers per cluster, number of fruits per cluster, number of fruits per plant, days to first flowering, days to $50 \%$ flowering, days to first harvest, days to last harvest, fruit length $(\mathrm{cm})$, fruit width $(\mathrm{cm})$, average fruit weight $(\mathrm{kg})$, fruit yield per plant $(\mathrm{kg})$, ascorbic acid content $(\mathrm{mg} / 100 \mathrm{~g})$, total phenol content $(\mathrm{mg} / 100 \mathrm{~g})$, shoot and fruit borer infestation (\%), cumulative wilt incidence (\%) and little leaf incidence (\%). The obtained data were subjected to RBD ANOVA following Panse and Sukhatme, 1985.

\section{Results and Discussion}

The analysis of variance (Table 2) revealed the significant differences among the genotypes for all the traits. Mean performance of all 36 brinjal genotypes were given in Table 3. 
Table.1 List of genotypes evaluated along with their sources

\begin{tabular}{|c|c|c|c|c|c|}
\hline S.No & IC No. & Source & S.No & IC No. & Source \\
\hline 1 & IC-136237 & $\begin{array}{l}\text { NBPGR, Regional } \\
\text { station, Hyderabad. }\end{array}$ & 19 & IC-135997 & $\begin{array}{l}\text { NBPGR, Regional } \\
\text { station, Hyderabad. }\end{array}$ \\
\hline 2 & IC-136297 & $\begin{array}{l}\text { NBPGR, Regional } \\
\text { station, Hyderabad. }\end{array}$ & 20 & IC-136300 & $\begin{array}{l}\text { NBPGR, Regional } \\
\text { station, Hyderabad. }\end{array}$ \\
\hline 3 & IC-136307 & $\begin{array}{l}\text { NBPGR, Regional } \\
\text { station, Hyderabad. }\end{array}$ & 21 & IC- 136248 & $\begin{array}{l}\text { NBPGR, Regional } \\
\text { station, Hyderabad. }\end{array}$ \\
\hline 4 & IC-136177 & $\begin{array}{l}\text { NBPGR, Regional } \\
\text { station, Hyderabad. }\end{array}$ & 22 & IC-212426 & $\begin{array}{l}\text { NBPGR, Regional } \\
\text { station, Hyderabad. }\end{array}$ \\
\hline 5 & IC-90178 & $\begin{array}{l}\text { NBPGR, Regional } \\
\text { station, Hyderabad. }\end{array}$ & 23 & IC-136309 & $\begin{array}{l}\text { NBPGR, Regional } \\
\text { station, Hyderabad. }\end{array}$ \\
\hline 6 & IC-136188 & $\begin{array}{l}\text { NBPGR, Regional } \\
\text { station, Hyderabad. }\end{array}$ & 24 & IC-136260 & $\begin{array}{l}\text { NBPGR, Regional } \\
\text { station, Hyderabad. }\end{array}$ \\
\hline 7 & IC-136196 & $\begin{array}{l}\text { NBPGR, Regional } \\
\text { station, Hyderabad. }\end{array}$ & 25 & IC-136231 & $\begin{array}{l}\text { NBPGR, Regional } \\
\text { station, Hyderabad. }\end{array}$ \\
\hline 8 & IC-136308 & $\begin{array}{l}\text { NBPGR, Regional } \\
\text { station, Hyderabad. }\end{array}$ & 26 & 5296 & $\begin{array}{l}\text { NBPGR, Regional } \\
\text { station, Hyderabad. }\end{array}$ \\
\hline 9 & IC-136290 & $\begin{array}{l}\text { NBPGR, Regional } \\
\text { station, Hyderabad. }\end{array}$ & 27 & IC-136096 & $\begin{array}{l}\text { NBPGR, Regional } \\
\text { station, Hyderabad. }\end{array}$ \\
\hline 10 & IC-136311 & $\begin{array}{l}\text { NBPGR, Regional } \\
\text { station, Hyderabad. }\end{array}$ & 28 & IC-136184 & $\begin{array}{l}\text { NBPGR, Regional } \\
\text { station, Hyderabad. }\end{array}$ \\
\hline 11 & IC-136546 & $\begin{array}{l}\text { NBPGR, Regional } \\
\text { station, Hyderabad. }\end{array}$ & 29 & IC-136311 & $\begin{array}{l}\text { NBPGR, Regional } \\
\text { station, Hyderabad. }\end{array}$ \\
\hline 12 & IC-144520 & $\begin{array}{l}\text { NBPGR, Regional } \\
\text { station, Hyderabad. }\end{array}$ & 30 & IC-136455 & $\begin{array}{l}\text { NBPGR, Regional } \\
\text { station, Hyderabad. }\end{array}$ \\
\hline 13 & IC-136093 & $\begin{array}{l}\text { NBPGR, Regional } \\
\text { station, Hyderabad. }\end{array}$ & 31 & IC-136148 & $\begin{array}{l}\text { NBPGR, Regional } \\
\text { station, Hyderabad. }\end{array}$ \\
\hline 14 & IC-136306 & $\begin{array}{l}\text { NBPGR, Regional } \\
\text { station, Hyderabad. }\end{array}$ & 32 & IC-154517 & $\begin{array}{l}\text { NBPGR, Regional } \\
\text { station, Hyderabad. }\end{array}$ \\
\hline 15 & IC-136450 & $\begin{array}{l}\text { NBPGR, Regional } \\
\text { station, Hyderabad. }\end{array}$ & 33 & Shyamala (C) & $\begin{array}{l}\text { Agricultural } \\
\text { Research Institute, } \\
\text { Hyderabad }\end{array}$ \\
\hline 16 & IC-203589 & $\begin{array}{l}\text { NBPGR, Regional } \\
\text { station, Hyderabad. }\end{array}$ & 34 & $\begin{array}{l}\text { Bhagyamathi } \\
\text { (C) }\end{array}$ & $\begin{array}{l}\text { Agricultural } \\
\text { Research Institute, } \\
\text { Hyderabad }\end{array}$ \\
\hline 17 & IC-136176 & $\begin{array}{l}\text { NBPGR, Regional } \\
\text { station, Hyderabad. }\end{array}$ & 35 & Gulabi (C) & $\begin{array}{l}\text { Agricultural } \\
\text { Research Institute, } \\
\text { Hyderabad }\end{array}$ \\
\hline 18 & IC-345333 & $\begin{array}{l}\text { NBPGR, Regional } \\
\text { station, Hyderabad. }\end{array}$ & & & \\
\hline
\end{tabular}

No: Accession number IC: Indigenous Collection; C: Check variety 
Table.2 ANOVA for yield and yield attributes in brinjal

\begin{tabular}{|c|c|c|c|c|}
\hline \multirow{2}{*}{$\begin{array}{l}\text { S. } \\
\text { No }\end{array}$} & \multirow[t]{2}{*}{ Character } & \multicolumn{3}{|c|}{ Mean sum of squares } \\
\hline & & $\begin{array}{c}\text { Replications } \\
(\mathbf{d f}=\mathbf{2})\end{array}$ & $\begin{array}{c}\text { Treatments } \\
(\mathbf{d f}=\mathbf{3 4})\end{array}$ & $\begin{array}{c}\text { Error } \\
(\mathrm{df}=68)\end{array}$ \\
\hline 1 & Plant height $(\mathrm{cm})$ & 3.36 & $69.52 * * *$ & 11.71 \\
\hline 2 & No. of branches per plant & 0.24 & $2.88 * *$ & 0.45 \\
\hline 3 & Days to first flowering & 0.32 & $51.06^{* *}$ & 1.07 \\
\hline 4 & Days to 50 percent flowering & 11.26 & $48.02^{* *}$ & 5.79 \\
\hline 5 & Number of flower clusters per plant & 1.46 & $15.56^{* *}$ & 1.44 \\
\hline 6 & Number of flowers per cluster & 0.016 & $0.46^{* *}$ & 0.05 \\
\hline 7 & Number of fruits per cluster & 2.98 & $0.37 * *$ & 0.05 \\
\hline 8 & Number of fruits per plant & 0.61 & $29.3 * *$ & 0.79 \\
\hline 9 & Days to first harvest & 5.06 & $91.74 * *$ & 5.77 \\
\hline 10 & Days to last harvest & 0.80 & $84.68 * *$ & 8.52 \\
\hline 11 & Fruit length $(\mathrm{cm})$ & 4.36 & $19.66^{* *}$ & 1.14 \\
\hline 12 & Fruit width $(\mathrm{cm})$ & 0.35 & $1.82 * *$ & 0.20 \\
\hline 13 & Average fruit weight $(\mathrm{kg})$ & 0.01 & $0.002 * *$ & 0.00 \\
\hline 14 & Fruit yield per plant (kg) & 0.001 & $0.52 * *$ & 0.03 \\
\hline 15 & Ascorbic acid content $(\mathrm{mg} / 100 \mathrm{~g})$ & 0.14 & $1.99 * *$ & 0.16 \\
\hline 16 & Total phenol content $(\mathrm{mg} / 100 \mathrm{~g})$ & 8.37 & $147.34 * *$ & 36.12 \\
\hline 17 & Shoot and fruit borer infestation (\%) & 4.96 & $23.81^{* *}$ & 2.28 \\
\hline 18 & Cumulative wilt incidence (\%) & 0.00 & 0.00 & 0.00 \\
\hline 19 & Little leaf incidence (\%) & 0.00 & 0.00 & 0.00 \\
\hline
\end{tabular}

* Significant at $\mathrm{P}=0.05$ level

$* *$ Significant at $\mathrm{P}=0.01$ level 
Table.3 Mean performance of yield and associated characters in thirty five genotypes of brinjal

\begin{tabular}{|c|c|c|c|c|c|c|c|c|c|c|c|c|c|c|c|c|c|c|c|c|}
\hline $\begin{array}{l}\dot{z} \\
\dot{n}\end{array}$ & 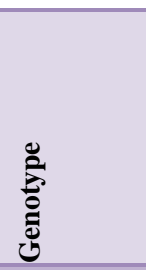 & 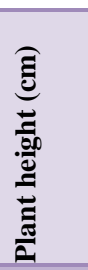 & 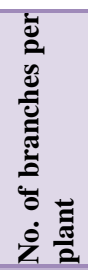 & 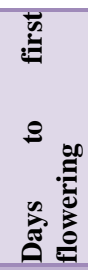 & 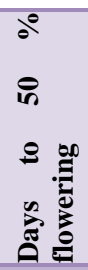 & 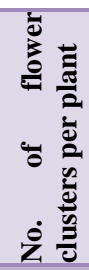 & 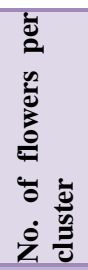 & 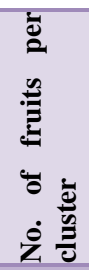 & 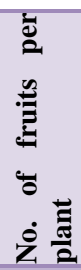 & 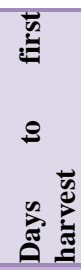 & 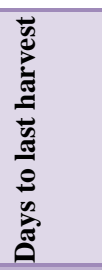 & 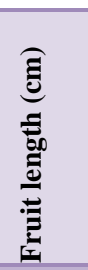 & 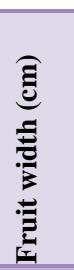 & 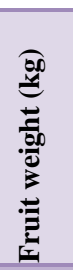 & 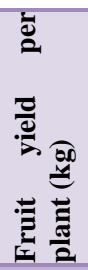 & 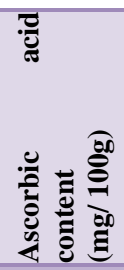 & 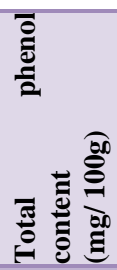 & 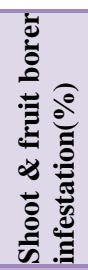 & 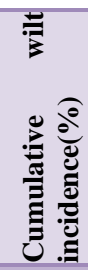 & 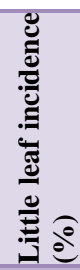 \\
\hline 1 & IC-136237 & 89.2 & 14.00 & 36.90 & 37.67 & 17.07 & 3.44 & 1.72 & 18.39 & 63.33 & 146.00 & 10.62 & 4.88 & 0.10 & 2.08 & 5.67 & 52.53 & 19.07 & 0.00 & 0.00 \\
\hline 2 & IC-136297 & 90.37 & 14.50 & 37.83 & 39.33 & 15.88 & 3.84 & 1.70 & 16.87 & 56.67 & 144.67 & 9.13 & 5.35 & 0.14 & 1.80 & 7.23 & 30.12 & 15.90 & 0.00 & 0.00 \\
\hline 3 & IC-136307 & 86.20 & 14.34 & 38.67 & 41.33 & 19.21 & 3.42 & 2.23 & 21.63 & 53.33 & 137.33 & 11.50 & 5.30 & 0.08 & 2.27 & 5.85 & 53.20 & 17.17 & 0.00 & 0.00 \\
\hline 5 & IC-90178 & 88.87 & 12.73 & 44.93 & 49.67 & 15.99 & 3.52 & 1.90 & 21.21 & 62.33 & 141.00 & 10.50 & 6.48 & 0.13 & 2.10 & 5.73 & 46.83 & 14.47 & 0.00 & 0.00 \\
\hline 6 & IC-136188 & 89.56 & 12.83 & 43.33 & 48.33 & 16.33 & 3.98 & 2.13 & 24.49 & 59.00 & 151.67 & 9.23 & 4.67 & 0.08 & 2.57 & 5.13 & 54.67 & 15.67 & 0.00 & 0.00 \\
\hline 7 & IC-136196 & 90.77 & 13.31 & 43.10 & 47.00 & 15.70 & 3.29 & 1.65 & 19.53 & 60.00 & 146.33 & 16.90 & 5.17 & 0.14 & 1.93 & 6.68 & 46.23 & 18.17 & 0.00 & 0.00 \\
\hline 8 & IC-136308 & 90.24 & 14.33 & 41.90 & 45.33 & 17.52 & 3.77 & 1.77 & 15.33 & 55.00 & 149.67 & 8.16 & 5.70 & 0.11 & 1.60 & 6.43 & 40.93 & 20.33 & 0.00 & 0.00 \\
\hline 9 & IC-136290 & 85.90 & 14.47 & 43.63 & 46.67 & 16.78 & 3.23 & 2.10 & 16.95 & 61.00 & 150.00 & 15.02 & 4.87 & 0.14 & 1.81 & 6.10 & 37.65 & 17.47 & 0.00 & 0.00 \\
\hline 10 & IC-136311 & 83.63 & 14.38 & 40.53 & 45.00 & 17.24 & 3.00 & 2.43 & 25.08 & 72.00 & 153.67 & 11.63 & 5.37 & 0.06 & 2.50 & 5.43 & 51.73 & 12.57 & 0.00 & 0.00 \\
\hline 12 & IC-144520 & 94.83 & 14.30 & 38.40 & 42.67 & 17.96 & 3.93 & 2.08 & 23.62 & 62.00 & 149.00 & 12.17 & 4.57 & 0.11 & 1.90 & 7.33 & 38.51 & 13.83 & 0.00 & 0.00 \\
\hline 13 & IC-136093 & 95.90 & 14.55 & 43.13 & 47.00 & 17.00 & 3.40 & 1.17 & 15.73 & 51.67 & 132.33 & 14.18 & 6.13 & 0.13 & 1.53 & 5.53 & 52.13 & 24.37 & 0.00 & 0.00 \\
\hline 14 & IC-136306 & 91.23 & 13.82 & 45.13 & 48.00 & 19.21 & 3.26 & 2.03 & 16.27 & 53.33 & 149.67 & 10.90 & 5.77 & 0.12 & 2.40 & 6.90 & 42.63 & 23.17 & 0.00 & 0.00 \\
\hline 15 & IC-136450 & 92.61 & 13.42 & 40.67 & 44.67 & 14.73 & 4.26 & 2.07 & 23.75 & 66.00 & 150.33 & 12.66 & 4.78 & 0.10 & 1.97 & 6.40 & 42.83 & 21.23 & 0.00 & 0.00 \\
\hline 16 & IC-203589 & 89.46 & 12.55 & 40.40 & 44.00 & 17.66 & 3.96 & 1.97 & 20.09 & 55.67 & 141.00 & 15.07 & 6.20 & 0.15 & 2.06 & 7.28 & 54.67 & 17.17 & 0.00 & 0.00 \\
\hline 17 & IC-136176 & 90.64 & 13.31 & 40.00 & 44.67 & 20.00 & 4.15 & 1.47 & 18.00 & 52.00 & 149.00 & 11.78 & 4.73 & 0.12 & 1.87 & 7.44 & 43.90 & 14.87 & 0.00 & 0.00 \\
\hline 18 & IC-345333 & 87.65 & 13.20 & 38.31 & 43.33 & 18.55 & 3.03 & 2.03 & 21.34 & 65.00 & 144.33 & 10.98 & 4.49 & 0.09 & 2.00 & 6.07 & 49.73 & 17.14 & 0.00 & 0.00 \\
\hline 19 & IC-135997 & 94.19 & 12.39 & 40.62 & 49.00 & 15.99 & 3.33 & 2.47 & 24.37 & 64.00 & 140.00 & 15.30 & 6.28 & 0.15 & 2.74 & 6.40 & 49.21 & 14.57 & 0.00 & 0.00 \\
\hline 20 & IC-136300 & 96.45 & 12.55 & 48.83 & 48.00 & 22.23 & 4.27 & 1.83 & 19.84 & 67.67 & 148.67 & 9.47 & 5.19 & 0.10 & 1.73 & 6.96 & 46.94 & 16.30 & 0.00 & 0.00 \\
\hline
\end{tabular}


Table.3 (Contd....)

\begin{tabular}{|c|c|c|c|c|c|c|c|c|c|c|c|c|c|c|c|c|c|c|c|c|}
\hline $\begin{array}{l}\dot{z} \\
\dot{m}\end{array}$ & 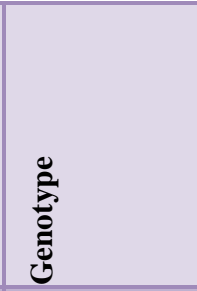 & 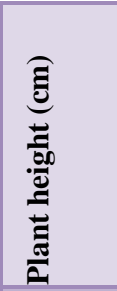 & 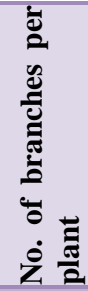 & 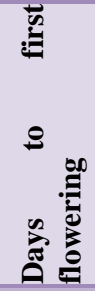 & 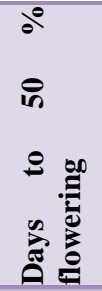 & 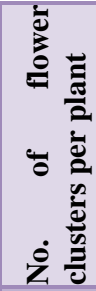 & 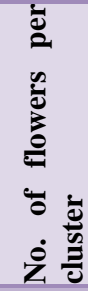 & 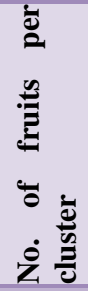 & 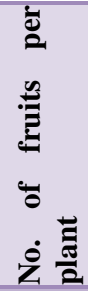 & 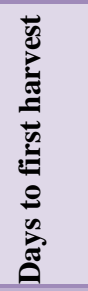 & 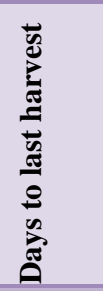 & 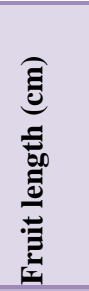 & 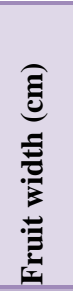 & 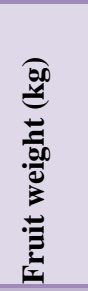 & 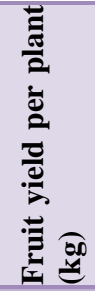 & 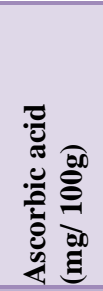 & 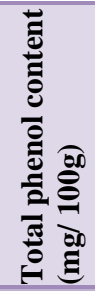 & 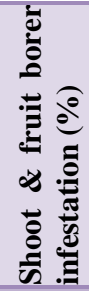 & 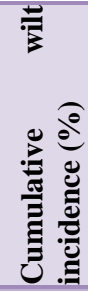 & 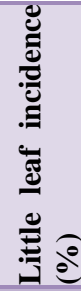 \\
\hline 21 & IC-136248 & 100.40 & $12 . \overline{24}$ & 43.33 & 47.33 & 17.92 & 3.27 & 1.10 & 17.54 & 55.33 & 143.33 & 14.38 & 4.06 & 0.12 & 2.30 & 6.27 & 40.79 & 13.78 & 0.00 & 0.00 \\
\hline 22 & IC-212426 & 101.36 & 12.55 & 39.77 & 41.33 & 18.70 & 4.22 & 1.85 & 22.37 & 62.33 & 147.00 & 9.80 & 3.87 & 0.09 & 2.77 & 4.93 & 55.00 & 14.89 & 0.00 & 0.00 \\
\hline 23 & IC-136309 & 100.11 & 13.15 & 38.07 & 40.67 & 15.77 & 3.99 & 2.03 & 21.49 & 68.33 & 148.33 & 11.47 & 3.17 & 0.03 & 2.47 & 6.73 & 40.80 & 12.93 & 0.00 & 0.00 \\
\hline 24 & IC- 136260 & 95.33 & 12.97 & 44.30 & 50.67 & 23.93 & 3.53 & 1.89 & 20.85 & 65.33 & 145.67 & 15.84 & 5.40 & 0.14 & 2.63 & 6.20 & 50.90 & 13.55 & 0.00 & 0.00 \\
\hline 25 & IC-136231 & 98.85 & 12.48 & 46.47 & 49.33 & 16.22 & 4.06 & 2.50 & 25.52 & 70.00 & 157.67 & 16.57 & 6.21 & 0.15 & 2.97 & 5.34 & 48.97 & 13.83 & 0.00 & 0.00 \\
\hline 26 & IC-136296 & 94.06 & 10.99 & 49.73 & 53.33 & 15.66 & 3.90 & 1.23 & 21.10 & 61.67 & 142.67 & 12.10 & 4.90 & 0.11 & 1.60 & 6.93 & 43.93 & 20.28 & 0.00 & 0.00 \\
\hline 27 & IC-136096 & 92.06 & 11.35 & 51.03 & 54.33 & 24.27 & 3.66 & 1.67 & 20.85 & 60.67 & 145.00 & 11.65 & 5.06 & 0.09 & 1.74 & 5.50 & 49.88 & 19.00 & 0.00 & 0.00 \\
\hline 28 & IC-136184 & 92.30 & 12.27 & 54.67 & 57.67 & 18.27 & 4.17 & 2.23 & 26.27 & 67.00 & 152.67 & 14.57 & 6.49 & 0.16 & 2.90 & 6.60 & 44.87 & 14.30 & 0.00 & 0.00 \\
\hline 29 & IC-136251 & 99.9 & 12.93 & 44.63 & 47.67 & 15.59 & 3.43 & 1.50 & 21.60 & 53.33 & 143.33 & 8.50 & 5.19 & 0.11 & 1.87 & 4.30 & 51.07 & 14.15 & 0.00 & 0.00 \\
\hline 30 & IC-136455 & 99.6 & 14.50 & 40.78 & 42.67 & 18.09 & 3.17 & 1.83 & 21.50 & 54.67 & 139.33 & 10.83 & 5.03 & 0.10 & 2.07 & 5.40 & 41.80 & 15.30 & 0.00 & 0.00 \\
\hline 31 & IC-136148 & 104.19 & 12.85 & 48.13 & 50.67 & 18.88 & 3.45 & 2.13 & 26.33 & 68.33 & 154.33 & 11.23 & 5.87 & 0.19 & 3.07 & 5.77 & 59.23 & 18.30 & 0.00 & 0.00 \\
\hline 32 & IC-154517 & 94.17 & 12.67 & 43.47 & 48.33 & 18.18 & 4.07 & 2.10 & 20.33 & 59.33 & 149.00 & 12.45 & 4.45 & 0.10 & 1.77 & 4.24 & 51.00 & 18.18 & 0.00 & 0.00 \\
\hline 33 & Shyamala & 94.77 & 12.29 & 45.17 & 50.00 & 17.07 & 3.47 & 2.01 & 23.71 & 61.33 & 152.33 & 8.39 & 4.27 & 0.09 & 1.93 & 5.27 & 49.20 & 14.60 & 0.00 & 0.00 \\
\hline 34 & Bhagyamathi & 94.8 & 12.27 & 44.65 & 50.33 & 21.03 & 3.27 & 2.32 & 26.20 & 64.67 & 150.00 & 8.30 & 4.03 & 0.07 & 1.87 & 6.21 & 54.27 & 16.88 & 0.00 & 0.00 \\
\hline 35 & Gulabi & 103.46 & 14.15 & 43.10 & 47.00 & 20.03 & 3.03 & 1.83 & 22.81 & 62.33 & 151.33 & 9.39 & 4.76 & 0.09 & 1.90 & 6.45 & 51.77 & 17.23 & 0.00 & 0.00 \\
\hline \multicolumn{2}{|c|}{ Grand Mean } & 93.35 & 13.20 & 43.02 & 46.75 & 18.11 & 3.65 & 1.89 & 21.08 & 60.87 & 146.62 & 11.92 & 5.10 & 0.11 & 2.12 & 6.10 & 46.79 & 16.66 & 0.00 & 0.00 \\
\hline \multicolumn{2}{|c|}{ SEm \pm} & 1.97 & 0.38 & 0.59 & 1.38 & 0.69 & 0.13 & 0.13 & 0.51 & 1.38 & 1.68 & 0.31 & 0.25 & 0.01 & 0.10 & 0.23 & 3.46 & 0.87 & 0.00 & 0.00 \\
\hline \multicolumn{2}{|c|}{$\mathrm{CV}(\%)$} & 3.66 & 5.08 & 2.40 & 5.14 & 6.64 & 6.47 & 12.73 & 4.22 & 3.94 & 1.99 & 8.96 & 8.75 & 14.05 & 8.41 & 6.55 & 12.84 & 9.07 & 0.00 & 0.00 \\
\hline \multicolumn{2}{|c|}{$\mathrm{CD}(\mathrm{P}=0.05)$} & 5.57 & 1.09 & 1.68 & 3.92 & 1.96 & 0.38 & 0.39 & 1.44 & 3.91 & 4.75 & 1.74 & 0.72 & 0.02 & 0.29 & 0.65 & 9.79 & 2.46 & 0.00 & 0.00 \\
\hline \multicolumn{2}{|c|}{ SE(d) } & 2.79 & 0.54 & 0.84 & 1.96 & 0.98 & 0.19 & 0.19 & 0.72 & 1.96 & 2.38 & 0.87 & 0.36 & 0.01 & 0.14 & 0.32 & 4.90 & 1.23 & 0.00 & 0.00 \\
\hline
\end{tabular}


Among the thirty five genotypes, IC-136148 $(104.19 \mathrm{~cm})$ showed maximum plant height, followed by IC-212426 $(101.36 \mathrm{~cm})$ and IC136248 (100.4) which was at par, while the minimum plant height $(83.63 \mathrm{~cm})$ was observed in IC-136311. The genotype IC136546 (15.00) recorded more number of branches per plant followed by IC-136093 (14.55) and IC-136297 (14.50) which was at par, whereas less number of branches per plant was recorded in IC-136296 (10.99).

Earliest flowering (36.55 days) was recorded in IC-136237 followed by IC-136546 (37.69 days), which was at par, while delayed flowering was recorded in IC-135184 (54.33 days). The genotype IC-136237 has taken only 37.67 days to reach 50 percent flowering stage, while IC-136184 was found to be late (57.67 days) among all the genotypes. The genotype IC-136096 recorded more number of flower clusters per plant followed by IC136260 (23.93), which was at par, whereas less number of flower clusters per plant was recorded in IC-136450.

The genotype IC-136300 recorded more number of flowers per cluster followed by IC136450 (4.26) and IC-212426 (4.22) which was at par, whereas less number of flowers per cluster was recorded in IC-136311. The genotype IC-136231 recorded more number of fruits per cluster, followed by IC-135997 (2.47), IC-136311 (2.43) and Bhagyamati (2.32) which was at par, whereas less number of fruits per cluster was recorded in IC136248. The more number of fruits per plant was recorded in IC-136148 (26.33) followed by IC-136184 (26.27), Bhagyamati (26.20) and IC-136231 (25.52) and less number of fruits per plant was observed in IC-136308 (15.33). Among the genotypes, less number of days taken for first harvest (51.67 days) was recorded in IC-136311, while more number of days (72.00) for first harvest was recorded in IC-136311. The genotype IC-136231 recorded maximum number of days for last harvest (157.67 days), whereas minimum number of days for last harvest was observed in IC-136093 (132.33 days).

Among the genotypes, highest fruit length $(16.90 \mathrm{~cm})$ was recorded in IC-136196, followed by IC-136231 (16.57 cm) which was on par and the genotype IC-136308 showed lowest fruit length $8.16 \mathrm{~cm}$ for this trait. Genotype IC-136309 showed lowest value of $3.17 \mathrm{~cm}$ and the genotype IC-136184 exhibited highest mean value of $6.49 \mathrm{~cm}$ for fruit width.

The highest fruit weight of $0.19 \mathrm{~kg}$ was recorded in IC-136148 followed by IC$136184(0.16 \mathrm{~kg})$ and IC-136231 (0.15 kg) and the lowest was observed in IC-136309 $(0.03 \mathrm{~kg})$. The genotype IC-136148 recorded the highest yield per plant $(3.07 \mathrm{~kg})$ followed by IC-136231 $(2.97 \mathrm{~kg}), \mathrm{IC}-136184(2.90 \mathrm{~kg})$, IC-135997 (2.74) and IC-136260 (2.63), which was at par, while the lowest yield was recorded in IC-136093 $(1.53 \mathrm{~kg})$.

Ascorbic acid content varied from $5.77 \mathrm{mg}$ $/ 100 \mathrm{~g}$ IC- 136148 to $7.44 \mathrm{mg} / 100 \mathrm{~g}$ and IC136176 with a general mean of $6.10 \mathrm{mg} / 100$ g. Total phenol content varied from 26.83 $\mathrm{mg} / 100 \mathrm{~g}$ (IC-136177) to $59.23 \mathrm{mg} / 100 \mathrm{~g}$ (IC136148) with a general mean of 46.79 $\mathrm{mg} / 100 \mathrm{~g}$. The lowest shoot and fruit borer infestation was observed in IC-136311 (12.57 $\%$ ) followed by IC-136309 (12.93\%), IC$136260(13.55 \%)$ and IC-136248 (13.78\%). In any selection programme, the mean performance of the genotypes for individual character serves as an important criterion for discarding the undesirable types. This indicates that germplasm studies may act as a potential source and offer scope for selection of high yielding genotypes with desirable horticultural attributes. Hence the present study, thrown a light on the potential of germplasm to act as genetic resources. 
In conclusion, based on the genetic studies, the genotypes five genotypes i.e. IC-136148, IC-212426, IC-136237, IC-136184 and IC136231 have been identified as promising genotypes with particular reference to the characters viz., plant height, days to first flowering, days to $50 \%$ flowering, days to last harvest, fruit length, fruit width, shoot and fruit borer infestation, average fruit weight, fruit yield per plant and ascorbic acid. Hence, these genotypes after multi location trials can be released for commercial cultivation. The genotype IC-136309 with tolerance to fruit and shoot borer infestation can be utilized in breeding programmes to develop high yielding coupled with resistant or used for developing hybrid varieties. IC-136176 for ascorbic acid, IC-136237 (Early flowering), IC-136196 (fruit length) possessed positive genes and hence, these germplasm can be used for pedigree breeding for further improvement.

\section{References}

Choudhary, B. 1976. Vegetable (4 ${ }^{\text {th }}$ edn.). National Book Trust, New Delhi. pp 5055

Dhankhar, B.S. and Singh, K. 1984. Path analysis for fruit yield and its components in brinjal (Solanum melongena L.). Haryana Journal of Horticultural Science. 12: 38-41.
Hanur, V.S. 2011. GM crops and centers of origin-a case study of $\mathrm{Bt}$ brinjal in India. Current Science. 100: 12851286.

Isshiki, K., Sen, G.B.A., Elder, D.E., Guerry, D. and Linneabach, A.J. 1994. Chromosome 9 deletion sporadic and familial melanomas in vivo. Oncogenea. 9: 1649-1653.

Kalloo, G., Banerjee, M. K., Singh, S.N. and M. Singh. (2002). Genetics of yield and its component characters in brinjal (Solanum melongena L.). Vegetable Science. 29: 24-26.

National Horticulture Data Base. 2014-15. National Horticulture Board, Ministry of Agriculture, Government of India.

Panse, V., G. and Sukhatme, P., V. 1985. Statistical Method for Agricultural Workers. Indian Council of Agricultural Research, New Delhi.

Vavilov, N.I. 1951. The phytogeographic basis of plant breeding. In: The origin, variation, immunity and breeding of cultivated plants. New York: The Ronald Press Company, 13-54 Translated by Chester KS.

Vinson, J.A., Hao, Y., Su, X. and Zubik, L. (1998). Phenol antioxidant quantity and quality in foods: vegetables. Journal of Agriculture Food Chemistry, 46: 36303634

\section{How to cite this article:}

Shiwangi Srivastava, P. Saidaiah, N. Shivraj and Ravinder Reddy, K. 2019. Yield and Quality Based Phenotyic Evaluation of Germplasm of Brinjal (Solanum melongena L.) Under SemiArid Conditions. Int.J.Curr.Microbiol.App.Sci. 8(07): 415-422. doi: https://doi.org/10.20546/ijcmas.2019.807.051 